$\begin{array}{ll}\text { Research Square } & \begin{array}{l}\text { Preprints are preliminary reports that have not undergone peer review. } \\ \text { They should not be considered conclusive, used to inform clinical practice, } \\ \text { or referenced by the media as validated information. }\end{array}\end{array}$

\title{
Evaluation of Temporomandibular Disorders Among Dental Students of Saudi Arabia Using Diagnostic Criteria/temporomandibular Disorders (DC/TMD) and Its Association With Biographic, Academic, and Psychosocial Parameters - A Cross-sectional Study
}

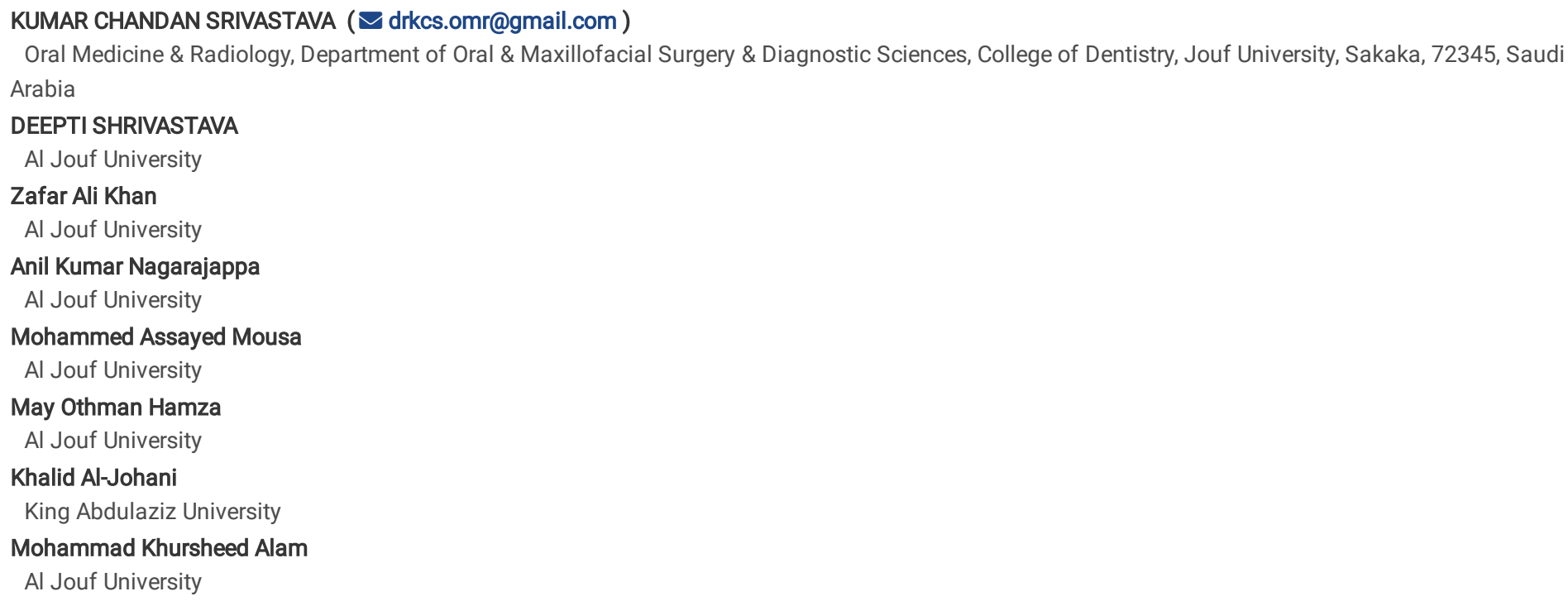




\section{Abstract}

Background: Temporomandibular disorders (TMD) are a board category of conditions arising from the various components of the temporomandibular joint (TMJ) complex. Bio-psychosocial model is the most accepted theory describing the etiopathogenesis of TMD. Dental students are vulnerable to psychological disorders including anxiety, depression and stress. Hence, the aim of the current study was to evaluate the TMD among dental students of various academic levels and explore the association of TMD with biographic, academic, and psychosocial parameters.

Methods: A total of 246 students of a dental school in Saudi Arabia were chosen for the study. After getting consent, all students were examined as per the diagnostic criteria/Temporomandibular disorders (DC/TMD) including components from axis-I and axis-II.

Results: The overall cross-sectional prevalence of TMD was found to be $36.99 \%$. Pain arising from the jaw, temple, and peri-auricular area was the most commonly reported symptom and elicited sign during examination. Among the pain-related TMD, myalgia was the commonest diagnosed condition, whereas disc displacement with reduction was found prevalent in intra-articular disorder category. Female (OR=1.94; $P=0.004)$, married (OR=1.74; $P=0.04)$, and students in clinical levels $(\mathrm{OR}=1.65 ; P=0.03)$ were shown to have significantly higher risk to develop TMD. Among the psychosocial parameters, parafunctional habits $(\mathrm{OR}=2.10 ; P<0.001)$ and anxiety $(\mathrm{OR}=1.55 ; P=0.04)$ are shown to increase risk of developing TMD. Students with any TMD reported to have significantly higher pain intensity $(\mathrm{OR}=1.68 ; P=0.01)$ and jaw functional limitations $(\mathrm{OR}=1.45 ; P=0.008)$. Conclusion: Dental students especially in the clinical levels were shown to poses higher risk of developing TMD, hence strategies such as academic counselling and objective evaluation via rubrics should be planned to modify the administration of the curriculum, training methods and evaluation process.

\section{Background}

The temporomandibular joint (TMJ) complex consists of bone, cartilage, muscles, ligaments, and associated neurovascular channels supplying to the structures [1]. The disorders arising from these structures are also complex in nature with varied presentations such as pain originating from bone and muscles; displacement of the cartilaginous disc, and degenerating conditions related to bony components [2]. As per the recent classification of TMD, the earlier mentioned conditions are known as pain related TMD and TMJ disorders respectively. Autoimmune conditions, trauma leading to fracture and dislocation of the joint also adds to the spectrum of conditions affecting the TMJ [3]. The presentation of pain related TMD and TMJ disorders do not remain restricted to the TMJ, but they are referred to neighbouring structures such as temples, areas inside and around the ears, forehead, and neck region [4]. They also bring limitations to the mandibular movements, thus causing distress in performing daily activities [5].

The aetiology of TMD is not clearly understood, but there are various predisposing factors which have been identified in various studies [6]. Initially, the cause of these conditions was considered as the biological component such as occlusion and its related disturbances [7]. With further research, studies have pointed the involvement of the psychological state of the patient [8]. At present, the bio-psychosocial model is considered as the most accepted concept that explains the complexity of the TMD [9]. With the modernization of the society, the psychological component has taken an upper hand, where conditions such as anxiety, depression, and distress have become more common. These conditions have been implicated as predisposing factors for various oral psychosomatic disorders, which are now being considered as lifestyle disorders. It includes conditions such as burning mouth syndrome, atypical odontalgia and TMD [10].

The complex nature of the TMD and its presentation also pose a problem in its evaluation, as there is diversity in presentation and subjectivity in recording the conditions. Considering these factors, in the year 1992, the research diagnostic criteria/temporomandibular disorder (RDC/TMD) was introduced [11, 12]. Initially, it was largely accepted by the international scientific community, but later concerns were raised regarding its sensitivity and specificity [13]. Thus a research group was made which organized various conferences and carried out large-scale epidemiological studies. With their efforts, modifications were brought to the criteria and the revised version was recognized as diagnostic criteria/temporomandibular disorder (DC/TMD) [3]. With the consensus over the involvement of biological and psychological components in the development of TMD, the bio-psychosocial model was agreed [14]. The same was adopted for its evaluation, where a dual-axis protocol was introduced through DC/TMD. In this system, the axis-I was made to assess the biological component and axis-II was designed to assess the psychological aspect. This is considered as the gold standard for evaluating TMD [3].

Earlier, studies employed RDC/TMD to evaluate the prevalence of TMD $[8,15,16]$. Later, the evaluating criterion was replaced with DC/TMD and studies were carried out in varied populations across different countries $[13,14,17]$. A common observation made in the past studies was the preponderance of TMD among females and in the younger age groups $[2,8,9,15]$. Previous studies have also pointed out the higher prevalence of TMD in university students, especially medical and dental undergraduates $[13,14,18,19]$. As we can see the complexity of the dental curriculum, it's demanding nature and challenging structure, the amount of stress, anxiety, and distress are bound to increase [20,21]. Hence, it will be important to evaluate the academic related parameters such as grade point average (GPA) and academic level influencing the TMD prevalence. Thus, the primary aim of the study was to assess the cross-sectional prevalence of TMD among dental students of varied academic level in Saudi Arabia. Furthermore it was intended to find a possible association between TMD and biographic, academic and psychosocial parameters in dental students.

\section{Methods Study identification}

A cross-sectional study was planned in a prospective manner. The study was conducted as per the guidelines of strengthening the reporting of observational studies in epidemiology (STROBE) [22]. The study was carried out during January to May 2018 after receiving the ethical approval from the ethics committee. Illustration about the sample 
The prospective study was conducted at a dental school in Saudi Arabia. In the current study, two academic parameters namely, academic level and academic performance (GPA) were considered. As per the five-year curriculum of the dental school, the students of the 1 st and 2 nd year are considered "preclinical", as they remain engaged in the dental skills laboratories. However, the students of the 3rd, 4th and 5th year learn and acquire skills in clinics and hence are considered in the "clinical" category. Based on the grade point average (GPA), the student's academic performance were categorized as low GPA (<3) and high GPA ( $\geq 3$ ). Both male and female students of $₫ 18$ years of age were included in the study. Subjects who had a current dental pain conditions and underwent treatment for such conditions were excluded from the study. Subjects who reported with a recognizable neurological disorder or are currently undergoing pharmacological treatment for any such conditions were also excluded from the study.

\section{Calibration of the Examiners:}

Based on the clinical experience in dealing with TMD and orofacial pain, four specialists were selected to be the examiners for the study. Irrespective of the clinical background, all examiners underwent a training session. During the session, they were provided with copies of the DC/TMD protocol of axis-I, axis-II and diagnostic decision tree. To enhance the understanding, all examiners saw instructional videos about the clinical examination available over the official webpage of the International Network for Orofacial pain \& Related disorders Methodology (INFORM). After they were acquainted with the theoretical aspect, they underwent clinical training with patients. The clinical training was extended until they gained sufficient expertise and came to an agreement about the protocol and diagnosis. The assessment of inter and intra examiner reliability was calculated by cronbac's alpha until we achieved a high level of agreement value of $₫ 0.80$.

\section{Data collection tools:}

The DC/TMD axis-I and axis-II protocol is considered to have high reliability index value and so considered as the gold standard [17]. For the same reason, we considered in the present study. The axis-I protocol includes a symptom questionnaire and an international version of the clinical examination. Based on the diagnostic criteria and reference to the decision tree, the diagnosis was made.

The standard DC/TMD symptom questionnaire comprises of 14 questions which helps to reveal the history, duration and weather any functional activity can modify the complaint by either aggravating or relieving it. The major symptoms which were enquired included pain (Q1-4), headache (Q5-7), jaw joint noise (Q8), and regarding the locking of the jaw while opening (Q9-12) and closing (Q13-14). With respect to the duration of complaint in the recent history, 30 days was considered as the benchmark while questioning about the complaint and its modifying factors [3]. The responses for all questions except the description of the pain were recorded in the dichotomous nature. The second part of the axis-l involved clinical examination, location of pain/headache, incisal relationship, midline pattern, mandibular movements, TMJ noise, joint locking, and muscle tenderness. All examination findings were performed for both sides of the jaw. As described in the diagnostic criteria, the combinations of positive responses from the history and examination components of DC/TMD axis-I, were designated a diagnosis for the particular subject [3].

The axis-II of DC/TMD is designed to assess the psychosocial parameters. In the current study, we undertook 5 standardized questionnaire namely graded chronic pain scale (GCPS), jaw limitation pain scale (JLPS), patient health questionnaire-9 (PHQ-9), generalized anxiety disorder (GAD) and oral behaviour checklist (OBC) [3]. These questionnaires are designed to assess different aspects of patient's psychosocial element.

\section{Study protocol and data collection:}

All subjects were clearly explained about the objectives of the study and were not influenced in any manner to participate in the study. Participants were asked to give written consent before starting the data collection. Initially, an educational camp was conducted at the dental school where all undergraduate students were encouraged to attend. The camp was extended for 2 weeks where students from all academic years were contacted sequentially. They were informed about the symptoms and presentation of TMD. All students who attended the camp were motivated to come for the TMD screening session. Students who attended the screening session were evaluated according the DC/TMD criteria. A total of 246 dental students participated in the study and underwent axis-I and axis-ll evaluation (Fig. 1).

The participants who gave voluntary consent were involved in the study. The study involved a 3 step protocol, where the first two steps involved the assessment of axis-I and in the last step, evaluation of axis-II was carried out. In the first step, the participants were given a symptom questionnaire. As per the axis-I protocol, students were subjected to symptom questionnaire followed by clinical examination. Based on the responses from axis-I and with the reference of decision tree, the specific diagnosis of TMD was made. Lastly, all students were subjected to five structured questionnaire as a part of axis-II evaluation.

\section{Data analysis:}

The distribution of data was done using number and percentages. For the inferential analysis, the univariate and multivariate analysis was performed for the variables, where $\mathrm{P}<0.05$ was considered as statistically significant. All data analysis was performed with SPSS v.21. For the purpose of diagnosis of the psychosocial parameters, pre-validated cut-off was used, as described previously [23-27]. While during inferential analysis of these parameters, as described previously, the scores were dichotomized as " 0 " absent and " 1 or $\geq 1$ " as present of psychosocial parameter [14].

\section{Results}

A total sample of 246 dental students across all academic levels from a dental school in Saudi Arabia was studied. The sample consisted majority of 20-25 years-aged subjects with male and female representing $55.69 \%$ and, $44.30 \%$ respectively. More than half of the subjects (63\%) belong to the clinical oriented academic levels. Considering the GPA, about $82.92 \%$ of the sample represented $\geq 3$ GPA, whereas only $17.07 \%$ of subjects has $<3$ (Table 1 ). 
Table 1: Descriptive characteristics of the study population

\begin{tabular}{|c|c|c|}
\hline Variable & Response & $f(\%)$ \\
\hline \multirow{3}{*}{ Age } & $<20$ Years & $64(26.01)$ \\
\hline & 20-25 Years & $134(54.47)$ \\
\hline & $₫ 25$ Years & $48(19.51)$ \\
\hline \multirow[t]{2}{*}{ Gender } & Male & $137(55.69)$ \\
\hline & Female & $109(44.30)$ \\
\hline \multirow[t]{2}{*}{ Marital Status } & Single & $190(77.23)$ \\
\hline & Married & $56(22.76)$ \\
\hline \multirow[t]{2}{*}{ Academic Year } & Preclinical & $91(36.99)$ \\
\hline & Clinical & $155(63)$ \\
\hline \multirow[t]{2}{*}{ Grade point average (GPA) } & $<3$ & $42(17.07)$ \\
\hline & $\geq 3$ & $204(82.92)$ \\
\hline
\end{tabular}

Based on the assessment of subjects with the criteria of DC/TMD axis-l, around $36.99 \%$ of subjects reported with at least one of the TMD. Pain arising from the areas such as jaw, temple, or in front of the ear was the most commonly (76.92\%) reported symptom followed by joint noise (30.76\%). On performing the clinical examination, pain/familiar pain was the most frequently recorded sign followed by alternations in the mandibular movement (64.83\%) (Table 2).

Table 2: Frequency distribution of symptoms \& signs reported by the subjects with TMD

\begin{tabular}{|lll|}
\hline Variable & Categories & Present $\mathrm{f}(\%)$ \\
\hline \multirow{3}{*}{ Overall Prevalence of TMD (at least one TMD) } & $91(36.99)$ \\
& Pain in Jaw, temple, in the ear or in front of ear & $70(76.92)$ \\
\cline { 2 - 3 } & Headache & $17(18.68)$ \\
\cline { 2 - 3 } & Joint Noise & $28(30.76)$ \\
\cline { 2 - 3 } & Jaw Locking & $5(5.49)$ \\
\cline { 2 - 3 } & Pain & $64(70.32)$ \\
\cline { 2 - 3 } & Headache & $15(16.48)$ \\
\cline { 2 - 3 } & Mandibular movement pattern & $59(64.83)$ \\
\cline { 2 - 3 } & Restricted mandibular movements & $4(4.39)$ \\
\cline { 2 - 3 } & Joint Noise & $17(18.68)$ \\
\cline { 2 - 3 } & Muscle Tenderness & $54(59.34)$ \\
\cline { 2 - 3 } & Joint Tenderness & $13(14.28)$ \\
\hline
\end{tabular}

By combining the positive findings of the symptom questionnaire and clinical examination, the TMD diagnosis namely, pain-related TMD, and intra-articular TMD were obtained. The distribution of individual conditions among the baseline variables are described in Table 3.

Table 3: Prevalence of TMD and frequency distribution of TMD diagnosis based on symptom questionnaire and clinical examination of Axis-I of DC/TMD among the variables 


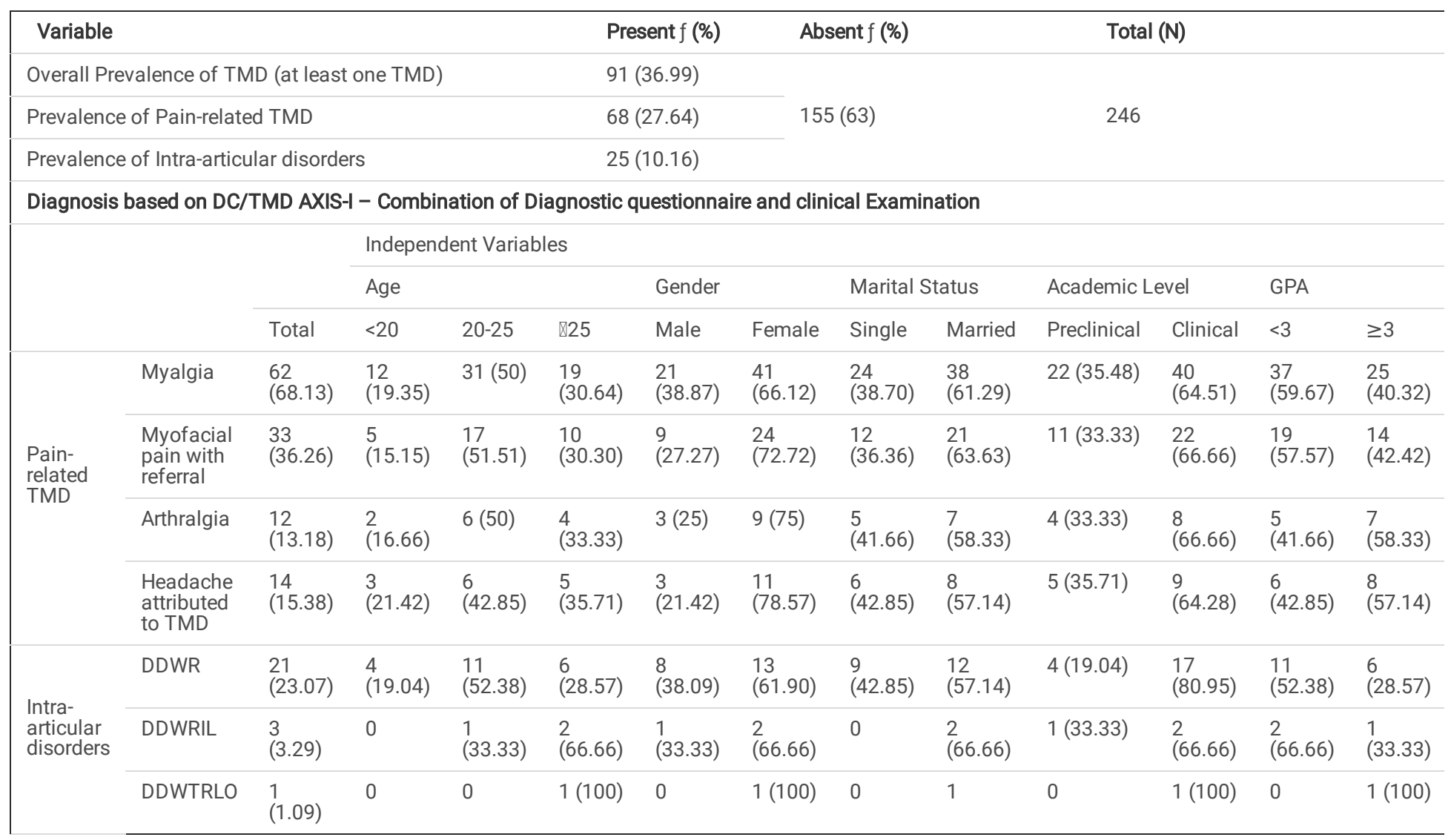

Note: DDWR - Disc displacement with reduction; DDWRIL - Disc displacement with reduction - With Intermittent locking; DDWTRLO - Disc displacement without reduction - without Limited opening

On multivariate analysis, female subjects were found to be at significantly ( $P=0.004)$ higher risk (1.94 times) for developing and TMD. Similarly, married subjects' poses 1.74 times $(P=0.03)$ higher risk of having TMD, in comparison to their unmarried counterparts. Additionally, students at the clinical level were reported to have increased risk $(P=0.04)$ than their preclinical contemporises (Table 4).

Table 4: Univariate and multivariate analysis assessing the risk indicators for developing TMD

\begin{tabular}{|c|c|c|c|c|c|}
\hline \multirow[t]{2}{*}{ Parameter } & & \multicolumn{2}{|c|}{ Univariate analysis } & \multicolumn{2}{|l|}{ Multivariate analysis } \\
\hline & & Odds Ratio (Cl) & $P$ value & Adjusted Odds Ratio (Cl) & $P$ value \\
\hline \multirow{3}{*}{ Age } & $<20$ Years & Reference & - & - & - \\
\hline & 20-25 Years & $1.55(0.65-1.96)$ & 0.16 & - & - \\
\hline & $\nabla 25$ Years & $1.86(0.74-2.01)$ & 0.29 & - & - \\
\hline \multirow[t]{2}{*}{ Gender } & Male & Reference & - & Reference & - \\
\hline & Female & $2.04(0.85-2.56)$ & $<0.001$ & $1.94(0.42-2.34)$ & 0.004 \\
\hline \multirow[t]{2}{*}{ Marital Status } & Single & Reference & - & Reference & - \\
\hline & Married & $1.95(0.62-2.45)$ & 0.01 & $1.74(0.35-2.10)$ & 0.04 \\
\hline \multirow[t]{2}{*}{ Academic Level } & Preclinical & Reference & - & Reference & - \\
\hline & Clinical & $1.84(0.54-2.78)$ & 0.002 & $1.65(0.35-2.24)$ & 0.03 \\
\hline \multirow[t]{2}{*}{ GPA } & $<3$ & $1.22(0.34-1.85)$ & 0.08 & - & - \\
\hline & $\geq 3$ & Reference & - & - & - \\
\hline
\end{tabular}

Note: $\mathrm{P}$ values mentioned in bold are statistically significant; $\mathrm{Cl}$ - Confidence interval 
On evaluating the psychological components, subjects with TMD were found to have a significantly higher risk to possess parafunctional habits ( $<<0.001)$ and anxiety $(P<0.04)$. Raised pain intensity level and altered jaw function are seen to be 1.68 times and 1.45 times higher in dental students with TMD in contrast to non-TMD students (Table 5).

Table 5: Univariate and multivariate analysis of Axis-II components of DC/TMD assessing the risk indicators for the development of any TMD

\begin{tabular}{|c|c|c|c|c|c|}
\hline \multirow[t]{2}{*}{ Parameter } & & \multicolumn{2}{|c|}{ Univariate analysis } & \multicolumn{2}{|l|}{ Multivariate analysis } \\
\hline & & Odds Ratio (Cl) & $P$ value & Adjusted Odds Ratio (Cl) & $P$ value \\
\hline \multirow[t]{2}{*}{ Pain Intensity-GCPS } & 0 & Reference & - & Reference & - \\
\hline & $\geq 1$ & $1.94(0.45-2.36)$ & $<0.001$ & $1.68(0.28-1.92)$ & 0.01 \\
\hline \multirow[t]{2}{*}{ Pain Function - JFLS-20 Item } & 0 & Reference & - & Reference & - \\
\hline & 1 & $1.68(0.30-2.15)$ & 0.006 & $1.45(0.10-1.85)$ & 0.008 \\
\hline \multirow[t]{2}{*}{ Anxiety - GAD $\quad$ (Sum Score 0-3) } & 0 & Reference & - & Reference & - \\
\hline & $\geq 1$ & $1.75(0.32-1.96)$ & 0.02 & $1.55(0.22-1.84)$ & 0.04 \\
\hline Depression & 0 & Reference & - & - & - \\
\hline PHQ-9 (Sum Score 0-4) & $\geq 1$ & $1.64(0.33-1.78)$ & 0.56 & - & - \\
\hline \multirow[t]{2}{*}{ Para function - Oral behavior checklist (OBC) Sum score $₫ 16=1$} & 0 & Reference & - & Reference & - \\
\hline & 1 & $2.42(0.48-3.10)$ & $<0.001$ & $2.10(0.35-2.56)$ & $<0.001$ \\
\hline
\end{tabular}

Note: $\mathrm{P}$ values mentioned in bold are statistically significant; $\mathrm{Cl}$ - Confidence interval

\section{Discussion}

The overall prevalence of TMD observed in our sample of dental students was $36.99 \%$. These disorders were found to occur significantly higher among female students in their clinical academic levels. On evaluating the axis-II, it was observed that anxiety and parafunctional habits significantly increase the risk of TMD.

TMD is a complex condition with a multifactorial etiology. The major clinical presentation of these disorders is pain in the orofacial region of non-odontogenic origin [12]. The present study was carried out on dental undergraduate students of Saudi Arabia. The majority of the studies had done in the past have used self-reported symptoms/questionnaires $[28,29]$ or $\operatorname{RDC} / \operatorname{TMD}[8,15]$, whereas the present study has an edge over others since we evaluated symptoms along with clinical examination using DC/TMD. Since TMD considerably affects daily functions, its early diagnosis, prevention, and therapeutic management are deemed necessary.

\section{Overall Prevalence of TMD}

In the past, studies done on the general population had observed around $25 \%$ of the population reporting symptoms of TMD, but very few were actually seeking treatment [30]. The present study reported 36.99\% prevalence of TMD, whereas a different study on Indian dental undergraduates reported a relatively less prevalence (30\%) [14].Similarly, in a study on medical undergraduates' prevalence (17\%) was even lesser [13]. A probable reason for the increased prevalence of TMD in the current study was the complexity level of the curriculum and the demanding nature of the study patterns followed in Saudi Arabia. Furthermore, dental students being more informed about the signs and symptoms associated with TMD, could have been more forthcoming in responding to the questions. In population-based studies $[9,16]$ the diagnostic criteria used was RDC/TMD and moreover, in a few studies [31] it was coupled with selfreported symptoms which were more subjective rather than objective.

\section{Association of TMD with biographic parameters}

A widely reported fact is that TMD are more prevalent in females [14, 32] and we too observed significant female preponderance with 1.94 times higher risk for developing TMD. However, previous study has reported a 2.3 times higher risk of TMD in female [32]. Another study with adolescent sample had found the similar gender variation [4]. The higher prevalence of TMD in females can be attributed to various gender oriented variations including hormonal, anatomical, behavioural and genetic [33]. It has been reported that the females during their reproductive period have higher levels of estrogens and progesterone. Under the influence of estrogens, the laxity of the ligament increases during the preovulatory phase which further gets attenuated with the movement of TMJ leading to irritation [34]. Anatomically, it has been observed that the TMJ translation is more stable in males compared to females because they posses larger mandibular condylar head, deep temporal fossa and the angle of the jaw is less obtuse. Additionally, the maxillary bones in males are longer, wider and thicker compare to females. Also, genetically researchers have found that patients with TMD disorders mostly experience pain in other area which is considered as co-morbidity and it is more common in female. Lastly, the coping strategies for stress have remained different among gender [34].

\section{Association of TMD with Academic parameters}


Another interesting observation made in the current study was the higher prevalence of TMD among the students belonging to the clinical levels with 1.65 times more likely chances to develop TMD. This can be understood by acknowledging the fact that these students have acquired theoretical knowledge about the TMD as a part of their curriculum. Hence, they can relate well to the symptoms and signs of TMD and so, thus responded to the questionnaire and clinical examination in a more responsible and informed manner. Inherent challenges, demanding patient care and apprehension about the carer are some of the concerns which can be attributed to the students in the clinical levels [19-21].

Stress, somatic distress, and depression are seen as important etiological risk factors for pain related TMDs, thus these conditions have shown to be commonly associated with psychological distress [35]. In the present study, married students were reported with 1.74 times higher risk to develop TMD compared to their unmarried counterpart. In accordance to our results, Blanco-Hungría A et al., reported higher prevalence of TMD among separated and divorced individuals followed by married and least among the single individual [36]. Contrary to this, Han W et al., reported single females to have more TMD disorders compared to married contemporaries [37]. Although married individuals could have additional emotional support, prevailing social, economic and professional demands could challenge their threshold, and offset the balance of personal and professional life. Hence, the married individuals find difficult to find a balance between the personal and professional life.

\section{Association of TMD with psychological parameters}

To assess the pain intensity, graded chronic pain scale (GCPS) was used in our study. It's a tested and reliable tool used only to measure the pain intensity but also quantifies the deleterious impact of pain while performing daily, recreational and social activities in the last 30 days [3]. In the present study, it was observed that the students with raised pain intensity are 1.68 times more likely to have any TMD. Pain is a subjective feeling and its threshold varies from person to person [38]. It is also noteworthy to mention that the pain behaviour undergoes modulation during its transmission to the higher centres and finally perception of pain at the supra-spinal levels is influenced by the psychological factors [39]. Although the pain attributed from the TMD disorders usually starts as a somatic disorder, but eventually progress towards the chronic pain, where the psychological components plays an important contributing role [40]. Likewise, in the current study the students with TMD were shown to have significantly higher pain intensity than with students with no TMD. Our results are in agreement with pervious study conducted with dental students of European continent [14].

The etiopathology of pain has been best described by the biopsychosocial model. According to this model, there is interplay between the biological and psychosocial component leading to the pain presentation [13]. In order to evaluate this, anxiety, depression and jaw functional limitation scale were assessed. The students with higher anxiety score were shown to have 1.55 times higher risk for developing TMD. Our observations restate the significance of the psychosocial component in the development of TMD. Student population generally seen to have higher anxiety levels compared to the adult population, as they remain in constantly challenging environment [20]. Additionally, previous studies have shown dental students to have higher level of stress and anxiety [41]. As an outcome of longstanding TMD, it was expected from the sample of dental students with TMD to show higher levels in JFLS. Conversely, subjects with high levels of JFLS were reported 1.45 times more likely to develop TMD.

The intention of including the oral behaviour checklist was to evaluate the impact of parafunctional habits. The checklist comprised of 21 questions pertaining to posture, motion and activities involving the oral musculoskeletal structures including the jaws and tongue. The assessment differentiated between the activities performed during sleep and awake state in the last month [3]. The students reported with parafunctional habits were found to have 2.10 times higher risk to develop TMD. Clenching/grinding teeth and chronic chewing gum were the most popular activities indicating a state of sub-conscious anxiety. This has shown to trigger muscle contraction leading to fatigue and eventually causing TMD [42].

\section{Strength \& Limitations}

In the current study we took representative sample from all academic years of dental school, in order to see impact of academic variables on the development of TMD. We employed the gold standard criteria - DC/TMD, for the assessment of TMD along with well calibrated examiners. Despite our encouraging results, there are few limitations in the study. Being a cross-sectional study design, the causal relationship between the variables cannot be established. Hence longitudinal studies with cohorts of all academic levels should be carried out. It will be also interesting to have multicenter study, so as to compare the pain behaviour.

\section{Conclusions}

The present study concludes that TMD are more prevalent in female dental students and students in clinical levels. Furthermore, pain was the most common sign and symptom associated with TMD disorder in dental students. Modifications in curriculum, training and evaluation methods could help reduce the occurrence of TMD in dental students.

\section{Declarations}

\section{Ethics approval and consent to participate}

All risks and benefits of the associated procedures were explained to all participants who signed informed consent before enrolment into the study. The study was approved by the institutional review board of Qaseem Private Colleges, college of Dentistry, Saudi Arabia with reference number EAC/812/2017 and was conducted following the Helsinki Declaration.

\section{Consent for publication}

Written informed consent for publication was obtained from the participants. 


\section{Availability of data and materials}

The datasets used and analysed during the current study are available from the corresponding author on reasonable request.

\section{Competing interests}

We declare no potential conflicts of interest with respect to the authorship or publication of this manuscript.

\section{Funding}

The current study is Self-funded

\section{Authors' contributions}

All authors contributed to this article. KCS, DS and KJ Conceptualized and developed methodology. KCS and DS collected the data and drafted of the manuscript. ZAK and AK analyzed the data. MAM and MH were involved in Validation, Visualization and revising the manuscript. KCS and MKA supervised the project, resources and revised the manuscript. All authors read and agreed with the content of the submitted manuscript.

\section{Acknowledgements}

None

\section{References}

1. Okeson J.P.O, Bell's Oral and Facial Pain. 7th ed. Chicago: Quintessence Pub Co Inc; 2014.

2. Katsikogianni E, Schweigert-Gabler S, Krisam J, Orhan G, Bissar A, Lux CJ, Schmitter M, Giannakopoulos NN. Diagnostic accuracy of the Diagnostic Criteria for Temporomandibular Disorders for children aged 8-12 years. Journal of Oral Rehabilitation. 2021 Jan;48(1):18-27.

3. Schiffman E, Ohrbach R, Truelove E, Look J, Anderson G, Goulet JP, List T, Svensson P. Diagnostic criteria for temporomandibular disorders (DC/TMD) for clinical and research applications: recommendations of the International RDC/TMD Consortium Network and Orofacial Pain Special Interest Group. Journal of oral \& facial pain and headache. 2014;28(1):6.

4. Graue AM, Jokstad A, Assmus J, Skeie MS. Prevalence among adolescents in Bergen, Western Norway, of temporomandibular disorders according to the DC/TMD criteria and examination protocol. Acta Odontologica Scandinavica. 2016 Aug 17;74(6):449-55.

5. Al-Khotani A, Naimi-Akbar A, Albadawi E, Ernberg M, Hedenberg-Magnusson B, Christidis N. Prevalence of diagnosed temporomandibular disorders among Saudi Arabian children and adolescents. The journal of headache and pain. 2016 Dec;17(1):41.

6. Sanders AE, Slade GD, Bair E, Fillingim RB, Knott C, Dubner R, Greenspan JD, Maixner W, Ohrbach R. General health status and incidence of first-onset temporomandibular disorder: the OPPERA prospective cohort study. The Journal of Pain. 2013 Dec 1;14(12):T51-62

7. Manfredini D, Perinetti G, Stellini E, Di Leonardo B, Guarda-Nardini L. Prevalence of static and dynamic dental malocclusion features in subgroups of temporomandibular disorder patients: Implications for the epidemiology of the TMD-occlusion association. Quintessence Int. 2015 Apr;46(4):341-9. doi: 10.3290/j.qi.a32986. PMID: 25386633.

8. de Melo Júnior PC, Aroucha JM, Arnaud M, Lima MG, Gomes SG, Ximenes R, Rosenblatt A, Caldas Jr AD. Prevalence of TMD and level of chronic pain in a group of Brazilian adolescents. PloS one. 2019 Feb 8;14(2):e0205874.

9. Wieckiewicz M, Grychowska N, Nahajowski M, Hnitecka S, Kempiak K, Charemska K, Balicz A, Chirkowska A, Zietek M, Winocur E. Prevalence and Overlaps of Headaches and Pain-Related Temporomandibular Disorders Among the Polish Urban Population. Journal of Oral \& Facial Pain \& Headache. 2020 Jan 1;34(1).

10. Shamim T. The psychosomatic disorders pertaining to dental practice with revised working type classification. The Korean journal of pain. 2014 Jan;27(1):16.

11. Dworkin SF, LeResche L. Research diagnostic criteria for temporomandibular disorders: review, criteria, examinations and specifications, critique. J Craniomandib Disord. 1992 Fall;6(4):301-55. PMID: 1298767.

12. Manfredini D, Guarda-Nardini L, Winocur E, Piccotti F, Ahlberg J, Lobbezoo F. Research diagnostic criteria for temporomandibular disorders: a systematic review of axis I epidemiologic findings. Oral Surgery, Oral Medicine, Oral Pathology, Oral Radiology, and Endodontology. 2011 Oct 1;112(4):453-62.

13. Jivnani HM, Tripathi S, Shanker R, Singh BP, Agrawal KK, Singhal R. A study to determine the prevalence of temporomandibular disorders in a young adult population and its association with psychological and functional occlusal parameters. Journal of Prosthodontics. 2019 Jan;28(1):e445-9.

14. Lövgren A, Österlund C, llgunas A, Lampa E, Hellström F. A high prevalence of TMD is related to somatic awareness and pain intensity among healthy dental students. Acta Odontologica Scandinavica. 2018 Aug 18;76(6):387-93.

15. Bertoli FM, Bruzamolin CD, Pizzatto E, Losso EM, Brancher JA, de Souza JF. Prevalence of diagnosed temporomandibular disorders: A cross-sectional study in Brazilian adolescents. PloS one. 2018 Feb 8;13(2):e0192254.

16. Progiante PS, Pattussi MP, Lawrence HP, Goya S, Grossi PK, Grossi ML. Prevalence of Temporomandibular Disorders in an Adult Brazilian Community Population Using the Research Diagnostic Criteria (Axes I and II) for Temporomandibular Disorders (The Maringá Study). Int J Prosthodont. 2015 NovDec;28(6):600-9. doi: 10.11607/ijp.4026. PMID: 26523719.

17. Skeie MS, Frid P, Mustafa M, Aßmus J, Rosén A. DC/TMD Examiner protocol: Longitudinal evaluation on interexaminer reliability. Pain Research and Management. 2018 Sep 26;2018.

Page $8 / 10$ 
18. Augusto VG, Perina KC, Penha DS, Santos DC, Oliveira VA. Temporomandibular dysfunction, stress and common mental disorder in university students. Acta ortopedica brasileira. 2016 Dec;24(6):330-3.

19. Ahuja V, Ranjan V, Passi D, Jaiswal R. Study of stress-induced temporomandibular disorders among dental students: An institutional study. National journal of maxillofacial surgery. $2018 \mathrm{Jul} ; 9(2): 147$.

20. Abdelsalam M, Rodriguez TE, Brallier L. Student and Faculty Satisfaction with Their Dental Curriculum in a Dental College in Saudi Arabia. International journal of dentistry. 2020 Apr 8;2020.

21. Cardall WR, Rowan RC, Bay C. Dental education from the students' perspective: curriculum and climate. Journal of dental education. 2008 May;72(5):6009.

22. Von Elm E, Altman DG, Egger M, Pocock SJ, Gøtzsche PC, Vandenbroucke JP. The Strengthening the Reporting of Observational Studies in Epidemiology (STROBE) statement: guidelines for reporting observational studies. Annals of internal medicine. 2007 Oct 16;147(8):573-7.

23. Von Korff M, Ormel J, Keefe FJ, Dworkin SF. Grading the severity of chronic pain. Pain. 1992 Aug 1;50(2):133-49.

24. Ohrbach R, Larsson P, List T. The jaw functional limitation scale: development, reliability, and validity of 8-item and 20-item versions. Journal of orofacial pain. 2008 Jul 1;22(3).

25. Kroenke K, Spitzer RL, Williams JB. The PHQ-9: validity of a brief depression severity measure. J Gen Intern Med. 2001 Sep;16(9):606-13. doi: 10.1046/j.1525-1497.2001.016009606.x. PMID: 11556941; PMCID: PMC1495268.

26. Ruiz MA, Zamorano E, García-Campayo J, Pardo A, Freire O, Rejas J. Validity of the GAD-7 scale as an outcome measure of disability in patients with generalized anxiety disorders in primary care. J Affect Disord. 2011 Feb;128(3):277-86. doi: 10.1016/j.jad.2010.07.010. Epub 2010 Aug 9. PMID: 20692043.

27. Ohrbach R, Markiewicz MR, McCall Jr WD. Waking-state oral parafunctional behaviors: specificity and validity as assessed by electromyography. European journal of oral sciences. 2008 Oct;116(5):438-44.

28. Habib SR, Al Rifaiy MQ, Awan KH, Alsaif A, Alshalan A, Altokais Y. Prevalence and severity of temporomandibular disorders among university students in Riyadh. The Saudi dental journal. 2015 Jul 1;27(3):125-30.

29. Al Hayek SO, Al-Thunayan MF, AlGhaihab AM, AlReshaid RM, Omair A. Assessing stress associated with temporomandibular joint disorder through Fonseca's anamnestic index among the Saudi physicians. Clinical and experimental dental research. 2019 Feb;5(1):52-8.

30. Murphy MK, MacBarb RF, Wong ME, Athanasiou KA. Temporomandibular joint disorders: A review of etiology, clinical management, and tissue engineering strategies. The International journal of oral \& maxillofacial implants. 2013 Nov;28(6):e393.

31. Alkhudhairy MW, Al Ramel F, Al Jader G, Al Saegh L, Al Hadad A, Alalwan T, Al Shaikh D, Al Bandar M. A self-reported association between temporomandibular joint disorders, headaches, and stress. Journal of International Society of Preventive \& Community Dentistry. 2018 Jul;8(4):371.

32. Bagis B, Ayaz EA, Turgut S, Durkan R, Özcan M. Gender difference in prevalence of signs and symptoms of temporomandibular joint disorders: a retrospective study on 243 consecutive patients. International journal of medical sciences. 2012;9(7):539.

33. Poveda Roda R, Bagán JV, Díaz Fernández JM, Hernández Bazán S, Jiménez Soriano Y. Review of temporomandibular joint pathology: Part I: Classification, epidemiology and risk factors. Medicina Oral, Patología Oral y Cirugía Bucal (Internet). 2007 Aug;12(4):292-8.

34. LeResche L, Saunders K, Von Korff MR, Barlow W, Dworkin SF. Use of exogenous hormones and risk of temporomandibular disorder pain. Pain. 1997 Jan 1;69(1-2):153-60.

35. Sójka A, Stelcer B, Roy M, Mojs E, Pryliński M. Is there a relationship between psychological factors and TMD?. Brain and behavior. 2019 Sep;9(9):e01360.

36. Blanco-Hungría A, Rodríguez-Torronteras A, Blanco-Aguilera A, Biedma-Velázquez L, Serrano-del-Rosal R, Segura-Saint-Gerons R. Influence of sociodemographic factors upon pain intensity in patients with temporomandibular joint disorders seen in the primary care setting. Medicina oral, patologia oral y cirugia bucal. 2012 Nov;17(6):e1034.

37. Han W, Kwon SC, Lee YJ, Park C, Jang EC. The associations between work-related factors and temporomandibular disorders among female full-time employees: findings from the Fourth Korea National Health and Nutrition Examination Survey IV (2007-2009). Annals of occupational and environmental medicine. 2018 Dec;30(1):1-8.

38. Christidis N, Lindström Ndanshau E, Sandberg A, Tsilingaridis G. Prevalence and treatment strategies regarding temporomandibular disorders in children and adolescents-A systematic review. Journal of oral rehabilitation. 2019 Mar;46(3):291-301.

39. Conti PC, Costa YM, Gonçalves DA, Svensson P. Headaches and myofascial temporomandibular disorders: overlapping entities, separate managements?. Journal of oral rehabilitation. 2016 Sep;43(9):702-15.

40. Graff-Radford SB, Bassiur JP. Temporomandibular disorders and headaches. Neurologic Clinics. 2014 May 1;32(2):525-37.

41. Basudan, N. Binanzan, A. Alhassan, “Depression, anxiety and stress in dental students," International journal of medical education vol. 8, pp. $179,2017$.

42. Florakis A, Fotinea SE, Yapijakis C. Subconscious temporomandibular dysfunction therapy: A new therapeutic approach for temporomandibular disorders. World Journal of Stomatology. 2014 Feb 20;3(1):10-8.

\section{Figures}




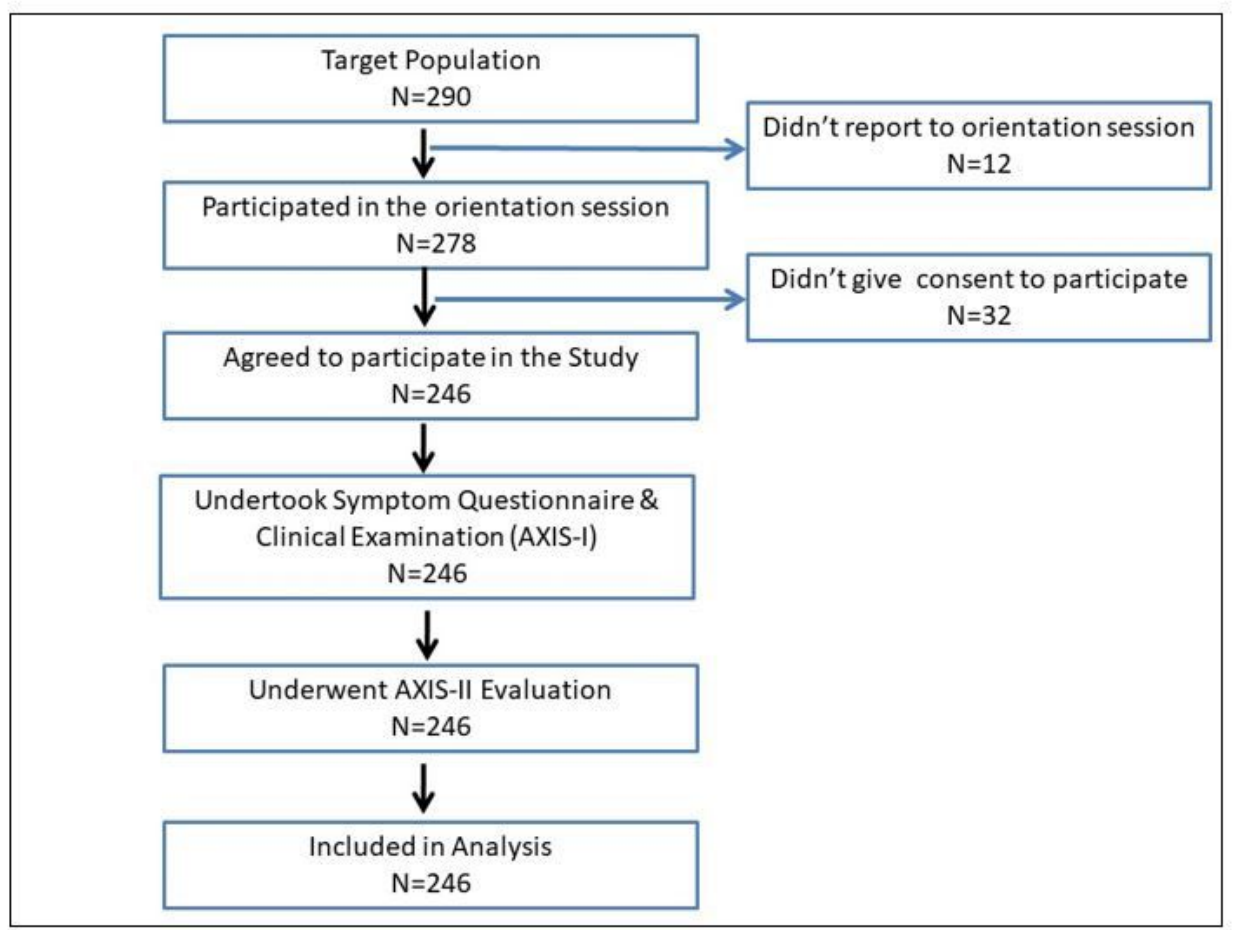

Figure 1

Flow chart of recruitment process of participants 